Article

\title{
Analysis of a Cantilevered Piezoelectric Energy Harvester in Different Orientations for Rotational Motion
}

\author{
Wei-Jiun Su ${ }^{1, * \mathbb{D}}$, Jia-Han Lin $^{2}$ and Wei-Chang $\mathrm{Li}^{2}$ (D) \\ 1 Department of Mechanical Engineering, National Taiwan University, No. 1, Sec. 4, Roosevelt Rd., \\ Taipei 10617, Taiwan \\ 2 Institute of Applied Mechanics, National Taiwan University, No. 1, Sec. 4, Roosevelt Rd., \\ Taipei 10617, Taiwan; r06543057@ntu.edu.tw (J.-H.L.); wcli@iam.ntu.edu.tw (W.-C.L.) \\ * Correspondence: weijiunsu@ntu.edu.tw
}

Received: 23 January 2020; Accepted: 19 February 2020; Published: 22 February 2020

\begin{abstract}
This paper investigates a piezoelectric energy harvester that consists of a piezoelectric cantilever and a tip mass for horizontal rotational motion. Rotational motion results in centrifugal force, which causes the axial load on the beam and alters the resonant frequency of the system. The piezoelectric energy harvester is installed on a rotational hub in three orientations-inward, outward, and tilted configurations-to examine their influence on the performance of the harvester. The theoretical model of the piezoelectric energy harvester is developed to explain the dynamics of the system and experiments are conducted to validate the model. Theoretical and experimental studies are presented with various tilt angles and distances between the harvester and the rotating center. The results show that the installation distance and the tilt angle can be used to adjust the resonant frequency of the system to match the excitation frequency.
\end{abstract}

Keywords: piezoelectric; energy harvesting; rotational motion; passive tuning

\section{Introduction}

Piezoelectric energy harvesting has attracted great attention in the past decade because of the demand for self-powered electronics, such as sensors [1-4], pacemakers [5,6], and mobile devices [7]. Several review papers have examined the development and evaluated the feasibility of using piezoelectric energy harvesting as an energy source for electronics [8,9]. A conventional piezoelectric energy harvester (PEH) has a cantilever structure with one or two piezoelectric patches attached for power generation. Many studies have been conducted to study the cantilevered PEH under base excitations. The mathematical model has been deeply discussed in the literature [10-14]. Although a cantilevered PEH has the advantage of simplicity, its resonant frequency needs to be properly tuned to match the excitation frequency in order to achieve high power output. In order to improve performance, different techniques have been adopted to achieve frequency tuning for piezoelectric energy harvesting from rectilinear excitations. Hu et al. [15] and Eichhorn et al. [16] utilized a screw to adjust the axial preload of a PEH to tune its resonant frequency. Magnetic force [17-19] has also been used to change the equivalent stiffness of PEHs. Alternatively to mechanical tuning, Moral et al. [20] and Brenes et al. [21] utilized the synchronized electrical charge extraction technique to perform frequency tuning for a strongly coupled piezoelectric energy harvester.

Besides scavenging kinetic energy from base excitations, PEHs can also be used to convert kinetic energy from rotational motion into electrical energy. Rotational PEHs can be categorized into two types. The first type contains a stator and a rotating hub, and these two components are coupled by magnetic force [22-26] or contact force [27]. When the rotating hub runs, reciprocating force will be 
generated to excite the PEH. Several studies [22-24] achieved energy harvesting from human motion by attaching a magnet on a freely rotating eccentric mass and relied on the magnetic force to pluck a piezoelectric cantilever with a tip magnet. Fu and Yeatman [25] discussed the influence of the arrangement of the rotating magnets and the beam on the performance of the PEH. Shu et al. [26] analyzed a rotary magnetic plucking PEH with a rectifier circuit. The other type of rotational energy harvesting is to mount a PEH on a horizontal rotating hub. As the hub rotates, the direction of the gravitational force varies with respect to the hub to excite the PEHs [28-42]. Although the excitation force caused by the gravity under rotational motion is similar to that caused by rectilinear excitations, the centrifugal force may affect the mechanical characteristics of the rotational PEHs. Gu et al. [28] and Hsu et al. [29] proposed an outwardly installed cantilevered PEH that used a tip mass at the free end for rotational energy harvesting. The resonant frequency of the PEH was passively tuned by the centrifugal force that changed with the rotational speed. Rui et al. [30] determined a design parameter for the outwardly installed rotational cantilevered PEH to optimize the self-tuning effect and achieve broadband harvesting. Guan and Liao [31] installed the PEH in the inward orientation and made the tip mass close to the rotating center to achieve a small centrifugal force. Instead of directly exciting the piezoelectric beam, $\mathrm{Gu}$ and Livermore [32] proposed a PEH composed of a driving beam with a tip mass and a driven piezoelectric beam. The driving beam was excited by the rotational motion and its tip hit the piezoelectric beam to generate energy. Wang et al. [33] proposed a self-tuning PEH for rotational motion. The resonant frequency could be adjusted by changing the effective length of the beam due to centrifugal force under rotational motion. Hsieh et al. [34] developed the theoretical model of a rotational PEH in outward, inward, and transverse configurations to examine their impact on the resonant frequency of the PEH. Instead of frequency tuning, Sadeqi et al. [35] proposed a broadband $\mathrm{PEH}$ by attaching a spring-mass system to a piezoelectric beam. By properly tuning the resonant frequency of the spring-mass system, the first two resonant frequencies of the PEH became close enough to form a broad bandwidth. Febbo et al. [36] and Ramírez et al. [37] utilized a multi-degree-of-freedom PEH composed of a multibeam structure to achieve low-frequency rotational energy harvesting. Bistable PEHs consisting of a piezoelectric cantilever beam and a pair of magnets were presented for rotational motion and showed the capability of broadband harvesting [38-41]. Zou et al. [42] developed a rotational PEH that consisted of two beams coupled by magnetic force to improve the bandwidth.

In the literature, a cantilevered rotational PEH in the inward and outward configurations at different installation distances has been investigated. However, there are no studies of the relationship between the installation angle and the dynamic characteristics of the rotational PEH. In this paper, we analyze the influence of the installation orientation of the rotational PEH on its performance. The theoretical model is derived and experimentally verified. This paper is organized as follows. The configurations of the PEH and its model are presented in Section 2, as well as the experimental set-up. The theoretical and experimental results are depicted in Section 3. Finally, conclusions are drawn in Section 4 .

\section{Materials and Methods}

Figure 1 shows the PEH in three different orientations: outward, inward, and tilted configurations. The orientation of the PEH influences the direction of the centrifugal force acting on the beam. Note that the outward and inward configurations are the tilted configuration when $\theta$ is $0^{\circ}$ and $180^{\circ}$, respectively. Therefore, the derivation of the model focused on the tilted configuration. The distance between the rotating center and the fixed end of the PEH is represented as $r_{0}$. 


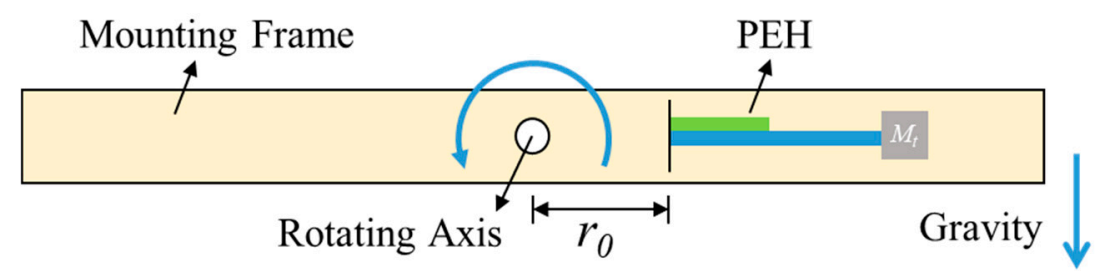

(a)

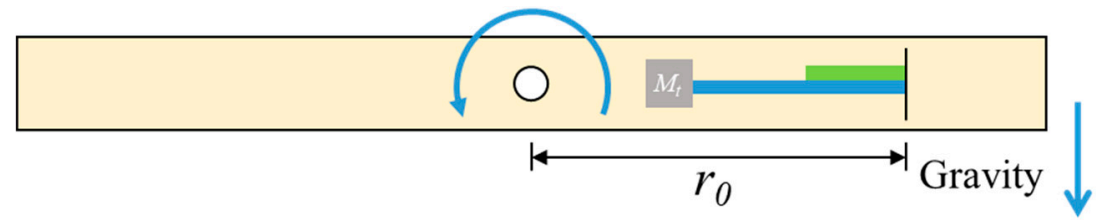

(b)

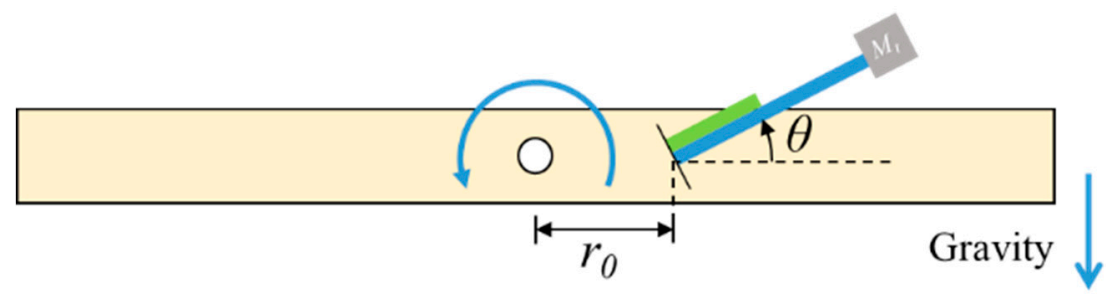

(c)

Figure 1. The piezoelectric energy harvester $(\mathrm{PEH})$ under rotational motion in different orientations: (a) the outward configuration, (b) the inward configuration, and (c) the tilted configuration.

\subsection{Modeling of the Proposed PEH}

The schematic of the PEH is shown in Figure 2. The PEH was a cantilevered beam with a partially covered piezoelectric layer. A tip mass was attached to the free end of the beam. The beam is was considered as a two-segment structure. The force and moment acting on the beam in the tilted configuration and an infinitesimal element of the beam is illustrated in Figure 3. The model is based on Euler-Bernoulli beam theory. Considering the inertial forces and the electromechanical coupling term, the equation of motion can be written as:

$$
\begin{gathered}
E I_{1} \frac{\partial^{4} w_{1}}{\partial x_{1}{ }^{4}}-P \frac{\partial^{2} w_{1}}{\partial x_{1}{ }^{2}}+m_{1} \frac{\partial^{2} w_{1}}{\partial t^{2}}-m_{1} \omega_{d}^{2} w_{1}+\varphi_{v} v(t)\left[\frac{d \delta\left(x_{1}\right)}{d x_{1}}-\frac{d \delta\left(x_{1}-L_{1}\right)}{d x_{1}}\right]=0 \\
E I_{2} \frac{\partial^{4} w_{2}}{\partial x_{2}{ }^{4}}-P \frac{\partial^{2} w_{2}}{\partial x_{2}{ }^{2}}+m_{2} \frac{\partial^{2} w_{2}}{\partial t^{2}}-m_{2} \omega_{d}^{2} w_{2}+m_{2} \omega_{d}^{2} r_{0} \sin \theta=0
\end{gathered}
$$

where the subscript indicates the segment number. The first segment was covered with a piezoelectric layer, while the second segment was not; $E I$ is the bending stiffness; $w$ is the transverse displacement of the beam; $m$ is the mass per unit length; $\omega_{d}$ is the rotational speed of the motor; $L_{1}$ is the length of the first segment; $r_{0}$ is the distance between the fixed end of the beam and the rotating axis; $\theta$ is the tilt angle of the beam; $P$ is the axial load due to the centrifugal force caused by the tip mass and can be expressed as:

$$
P=M_{t} \omega_{d}^{2}\left(r_{0} \cos \theta+L\right)
$$

where $M_{t}$ is the tip mass; and $L$ is the total length of the beam. 


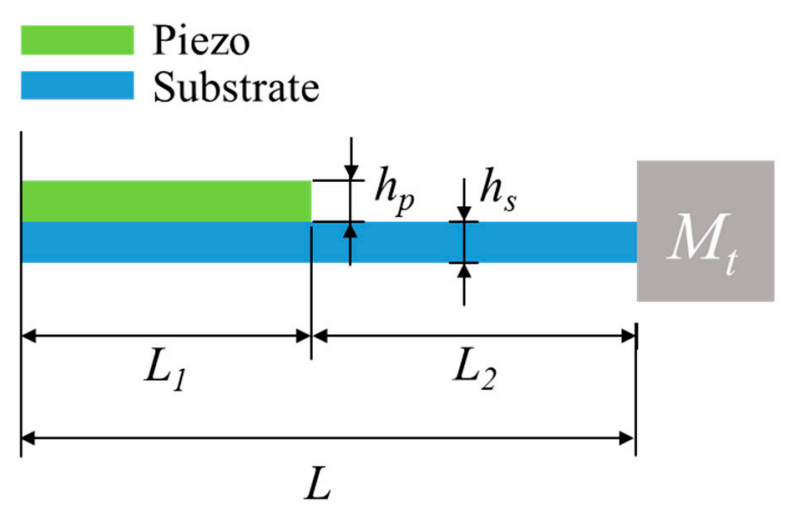

Figure 2. Schematic of the PEH.

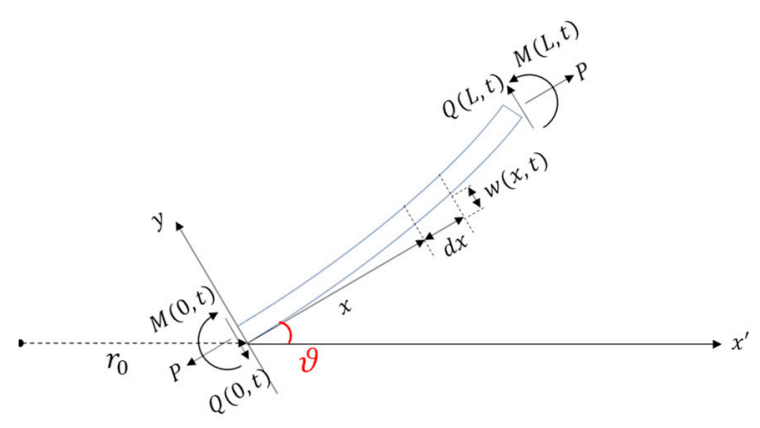

(a)

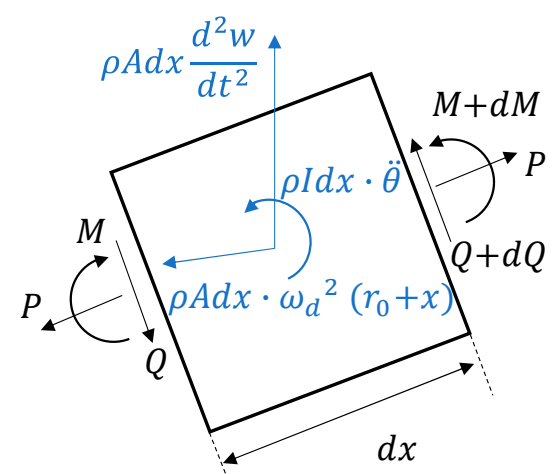

(b)

Figure 3. Force diagrams of (a) the whole beam (b) an infinitesimal element.

The boundary and continuous conditions of the structure are listed below:

$$
\begin{gathered}
w_{1}(0, t)=0 \\
w_{1}^{\prime}(0, t)=0 \\
w_{1}\left(L_{1}, t\right)=w_{2}(0, t) \\
w_{1}^{\prime}\left(L_{1}, t\right)=w_{2}^{\prime}(0, t) \\
E I_{1} w_{1}^{\prime \prime}\left(L_{1}, t\right)=E I_{2} w_{2}^{\prime \prime}(0, t) \\
E I_{1} w_{1}^{\prime \prime \prime}\left(L_{1}, t\right)=E I_{2} w_{2}^{\prime \prime \prime}(0, t) \\
E I_{2} w_{2}^{\prime \prime}\left(L_{2}, t\right)=0 \\
E I_{2} w_{2}^{\prime \prime \prime}\left(L_{2}, t\right)-P \cdot w_{2}^{\prime}\left(L_{2}, t\right)+M_{t} \omega_{d}^{2} w_{2}\left(L_{2}, t\right)-M_{t} \ddot{w}_{2}\left(L_{2}, t\right)=0
\end{gathered}
$$

The transverse motion of the beam can be rewritten as:

$$
w_{k}\left(x_{k}, t\right)=\sum_{i=1}^{\infty} \phi_{k i}\left(x_{k}\right) \eta_{i}(t)
$$

where $\phi_{k i}(x)$ is the mode shape function of the $k$-th segment and the $i$-th mode; $\eta_{i}(t)$ is the temporal function. In this study, only the first mode was considered. The mode shape function can be further represented as:

$$
\phi_{1}(x)=C_{1} \sin \left(\alpha_{1} x\right)+C_{2} \cos \left(\alpha_{1} x\right)+C_{3} \sinh \left(\alpha_{2} x\right)+C_{4} \cosh \left(\alpha_{2} x\right)
$$




$$
\phi_{2}(x)=C_{5} \sin \left(\alpha_{3} x\right)+C_{6} \cos \left(\alpha_{3} x\right)+C_{7} \sinh \left(\alpha_{4} x\right)+C_{8} \cosh \left(\alpha_{4} x\right)
$$

where

$$
\begin{aligned}
& \alpha_{1}^{2}=\frac{-K_{1}^{2}+\sqrt{K_{1}^{4}+4 \beta_{1}^{4}}}{2} \\
& \alpha_{2}^{2}=\frac{K_{1}^{2}+\sqrt{K_{1}^{4}+4 \beta_{1}^{4}}}{2} \\
& \alpha_{3}^{2}=\frac{-K_{2}^{2}+\sqrt{K_{2}^{4}+4 \beta_{2}^{4}}}{2} \\
& \alpha_{4}^{2}=\frac{K_{2}^{2}+\sqrt{K_{2}^{4}+4 \beta_{2}^{4}}}{2}
\end{aligned}
$$

and

$$
\begin{gathered}
K_{k}^{2}=\frac{M_{t} \omega_{d}^{2}\left(r_{0} \cos \theta+L\right)}{E I_{k}}, k=1,2 \\
\beta_{k}^{4}=\frac{m_{k}\left(\omega_{n}^{2}+\omega_{d}^{2}\right)}{E I_{k}}, k=1,2
\end{gathered}
$$

where $\omega_{n}$ is the resonant frequency of the beam.

The orthogonality condition was used to mass-normalize the mode shape function:

$$
\int_{0}^{L_{1}} \phi_{1 m} m_{1} \phi_{1 n} d x_{1}+\int_{0}^{L_{2}} \phi_{2 m} m_{2} \phi_{2 n} d x_{2}+\phi_{2 m}\left(L_{2}\right) M_{t} \phi_{2 n}\left(L_{2}\right)=\delta_{m n}
$$

Therefore, the equations of motion can be expressed as:

$$
\frac{d^{2} \eta(t)}{d t^{2}}+2 \zeta_{0} \omega_{n} \frac{d \eta(t)}{d t}+\omega_{n}^{2} \eta(t)+\Theta V(t)=f(t)
$$

where $\zeta_{0}$ is the damping ratio of the system and $f(t)$ is the normalized coefficient for excitation force caused by the gravitational force of the tip mass:

$$
f(t)=-\phi_{2}\left(L_{2}\right) \cdot M_{t} g \cos \omega_{d} t
$$

The piezoelectric layer can be considered as a capacitor in parallel with a current source so the electromechanical coupling equation of the circuit can be expressed as:

$$
-\Theta \dot{\eta}(t)+C_{p} \dot{V}_{p}(t)+\frac{1}{R} V_{p}(t)=0
$$

where $V_{p}$ is the voltage across the resistive load $R ; C_{p}$ is the capacitance of the piezoelectric layer. The coupling coefficient can be written as:

$$
\Theta=\int_{0}^{L_{1}} e_{31} b_{p} h_{p c} \frac{\partial^{3} w_{1}}{\partial x_{1}^{2} \partial t} d x_{1}
$$

\subsection{Experimental Setup}

The experimental setup for the rotational tests of the PEH is depicted in Figure 4. A variable-speed drive (Oriental Motor BMUD200-A) was used to control the speed of the motor (Oriental Motor BLM5200-A), which drove a mounting frame. The PEH was fixed on the mounting frame by a clamp. The clamp was screwed on the frame, which allowed the clamp to be installed at different positions 
and in different orientations. The PEH was composed of a cantilever beam covered with an MFC (Macro-Fiber Composite) patch (M-2807-P2) and a tip mass was attached to the end of the beam. The MFC patch was more flexible than piezoelectric ceramics, so it was more suitable for the PEH with large deflection. The distance between the fixed end of the PEH and the rotating axis could be adjusted. The titled angle of the PEH could also be tuned to see its influence on the performance of the PEH. An oscilloscope (Rigol DS1104Z) was used to measure the voltage output of the PEH.

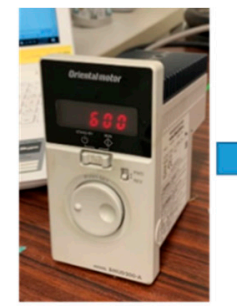

Variable Speed Drive

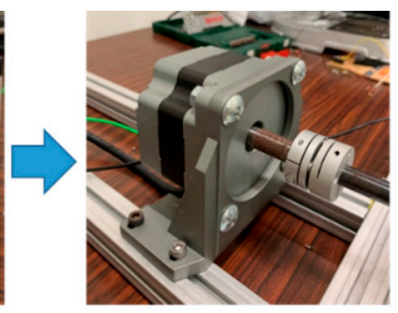

Servo Motor

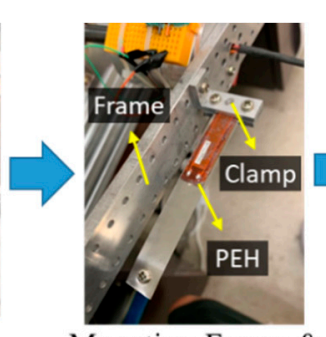

Mounting Frame \& $\mathrm{PEH}$

Figure 4. Experimental setup.

\section{Results and Discussion}

The rotational PEH was first installed in the outward configuration $\left(\theta=0^{\circ}\right)$. The corresponding parameters are listed in Table 1. Figure 5 shows the resonant frequency of the PEH installed with different $r_{0}$. In the outward orientation, the centrifugal force resulted in tensile force on the beam so the resonant frequency increased as the driving frequency rose. The matching frequencies, which are located at intersections between the solid lines and the dashed line, indicate that the resonant frequency of the PEH matched the driving frequency. Note that the resonant frequency increased as $r_{0}$ was enlarged under the same driving frequency. That is because the centrifugal force caused by the tip mass was enhanced when $r_{0}$ increased. The slope of the curve became steeper when $r_{0}$ increased. Figure 6 depicts the simulation and experimental results of the voltage responses. The experimental results match the simulation well. The numerical peak voltage and resonant frequency were close to the experimental results. As predicted by the model, the experimental result showed that the matching frequency rose when $r_{0}$ was enlarged.

Table 1. Parameters of the PEH in the outward configuration.

\begin{tabular}{ccc}
\hline Symbol & Description & Value \\
\hline$L$ & Length (beam) & $75 \mathrm{~mm}$ \\
$b_{s}$ & Width (beam) & $12.7 \mathrm{~mm}$ \\
$h_{s}$ & Thickness (beam) & $0.1 \mathrm{~mm}$ \\
$\rho_{s}$ & Density (beam) & $7930 \mathrm{~kg} / \mathrm{m}^{3}$ \\
$E_{s}$ & Young's modulus (beam) & $193 \mathrm{GPa}$ \\
$L_{1}$ & Length (MFC) & $28 \mathrm{~mm}$ \\
$b_{p}$ & Width (MFC) & $7 \mathrm{~mm}$ \\
$h_{p}$ & Thickness (MFC) & $0.3 \mathrm{~mm}$ \\
$\rho_{p}$ & Density (MFC) & $5440 \mathrm{~kg} / \mathrm{m}^{3}$ \\
$E_{p}$ & Young's modulus (MFC) & $30.336 \mathrm{GPa}$ \\
$M_{t}$ & Tip mass & $3.04 \mathrm{~g}$ \\
$R$ & Load resistance & $1 \mathrm{M} \Omega$ \\
\hline
\end{tabular}




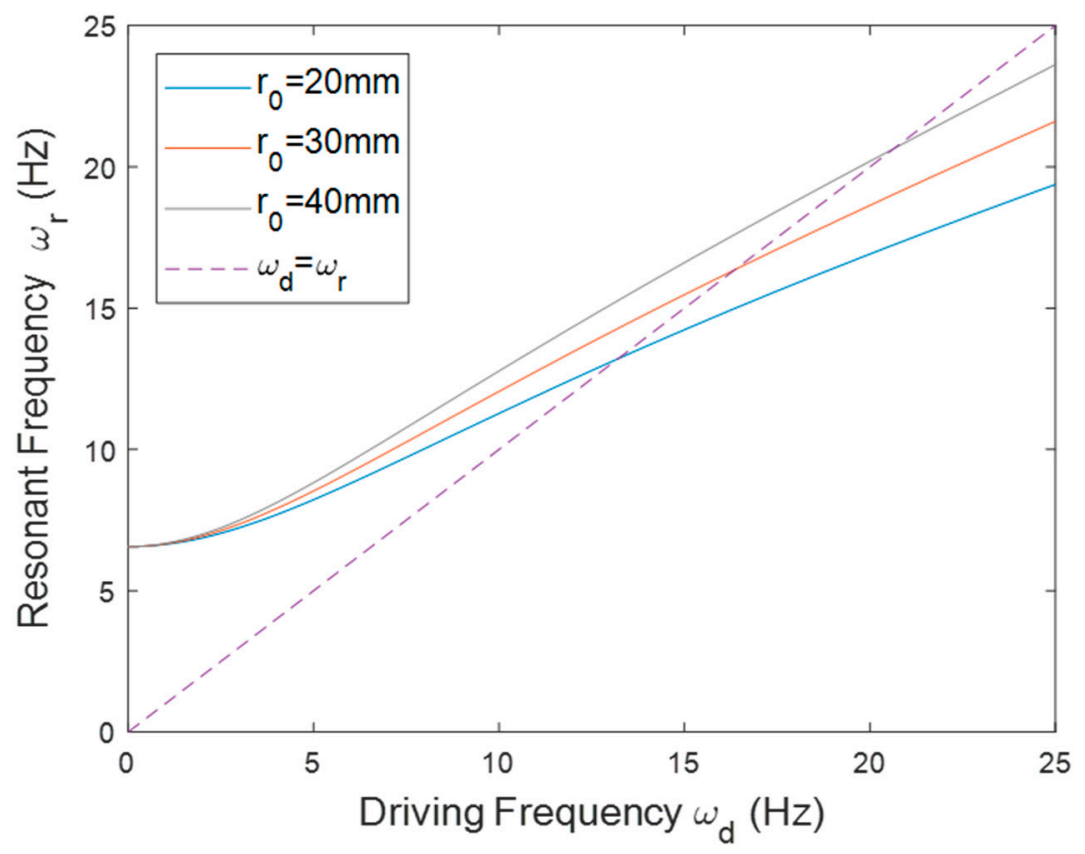

Figure 5. The resonant frequency of the PEH in the outward orientation with different $r_{0}$.

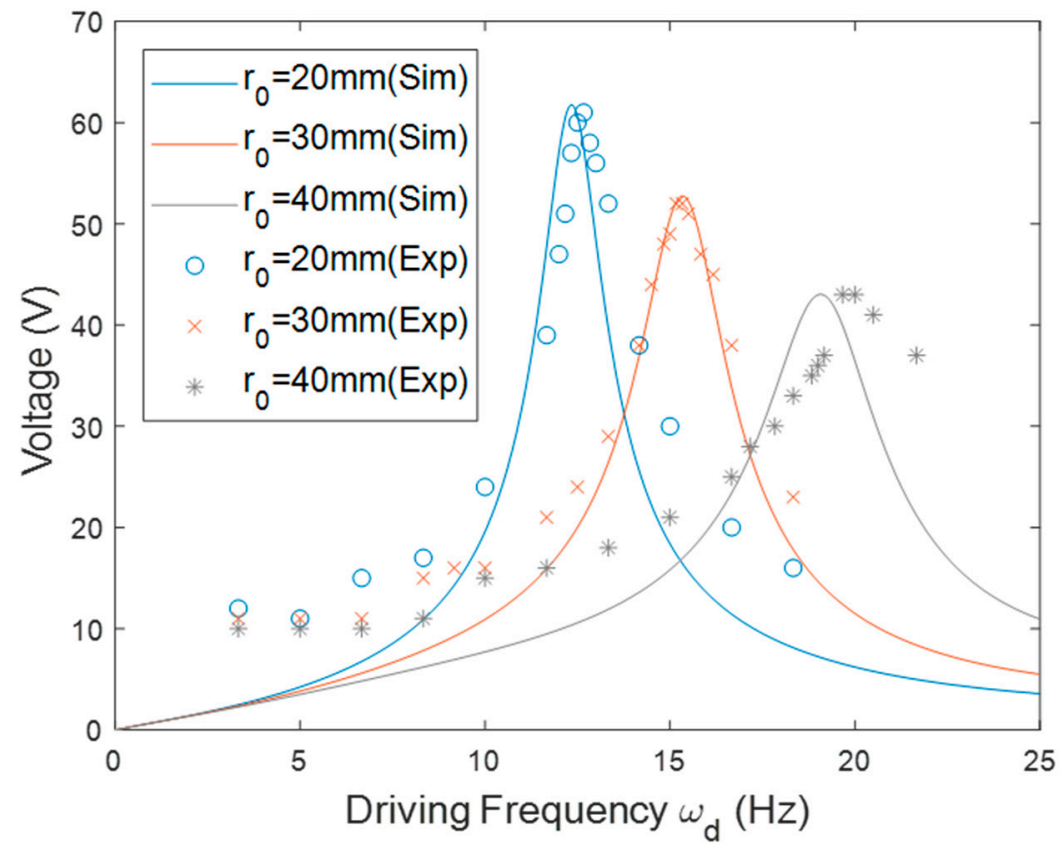

Figure 6. The simulation and experimental voltage responses of the $\mathrm{PEH}$ in the outward orientation with different $r_{0}$.

For the inward configuration, the beam was slightly shortened from 75 to $72 \mathrm{~mm}$ to prevent the excessive amplitude of the tip displacement, since a longer beam would otherwise lead to a larger tip displacement. Figure 7 shows the resonant frequency of the PEH with different $r_{0}$. The resonant frequency decreased as the driving frequency increased because the centrifugal force worked as compressive force on the beam to lower its stiffness when the beam was placed in the inward orientation. The slope of the curve became steeper when $r_{0}$ increased. The matching frequency decreased when $r_{0}$ was enlarged. Figure 8 depicts the frequency responses of the PEH with different $r_{0}$. The resonant frequency was raised as $r_{0}$ decreased in both the simulation and experimental results. 


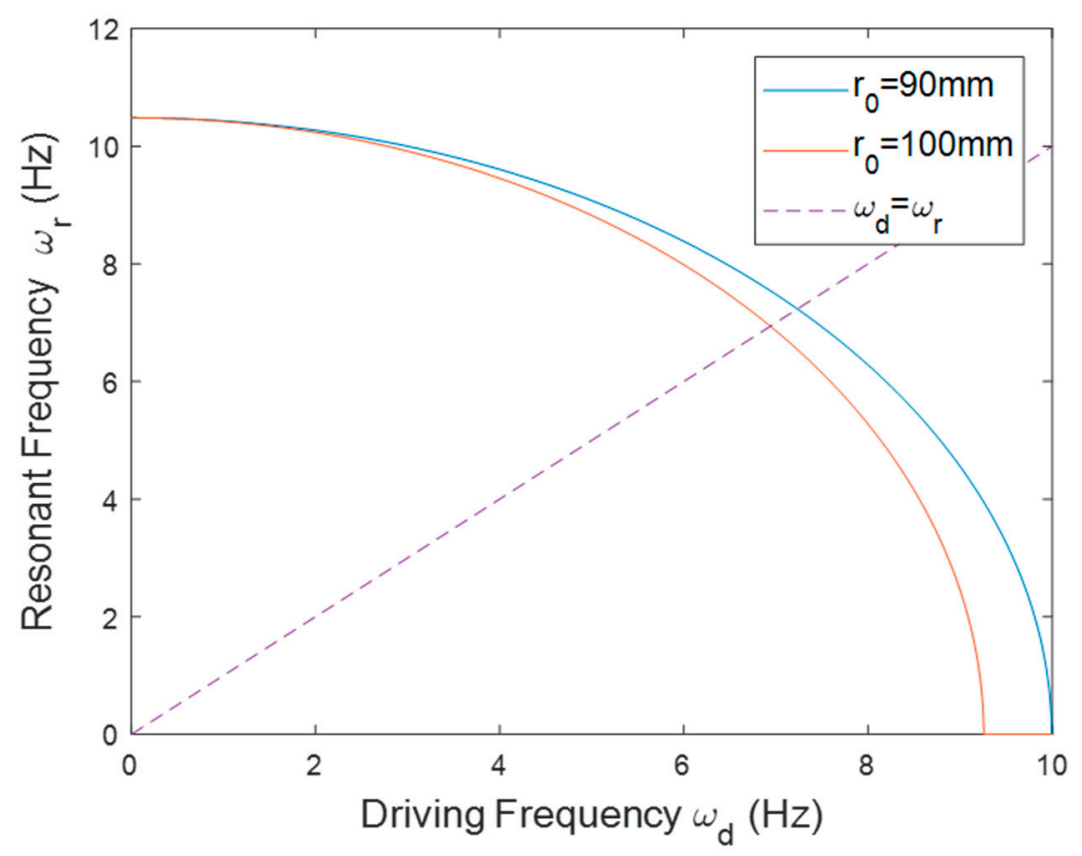

Figure 7. The resonant frequency of the PEH in the inward orientation with different $r_{0}$.

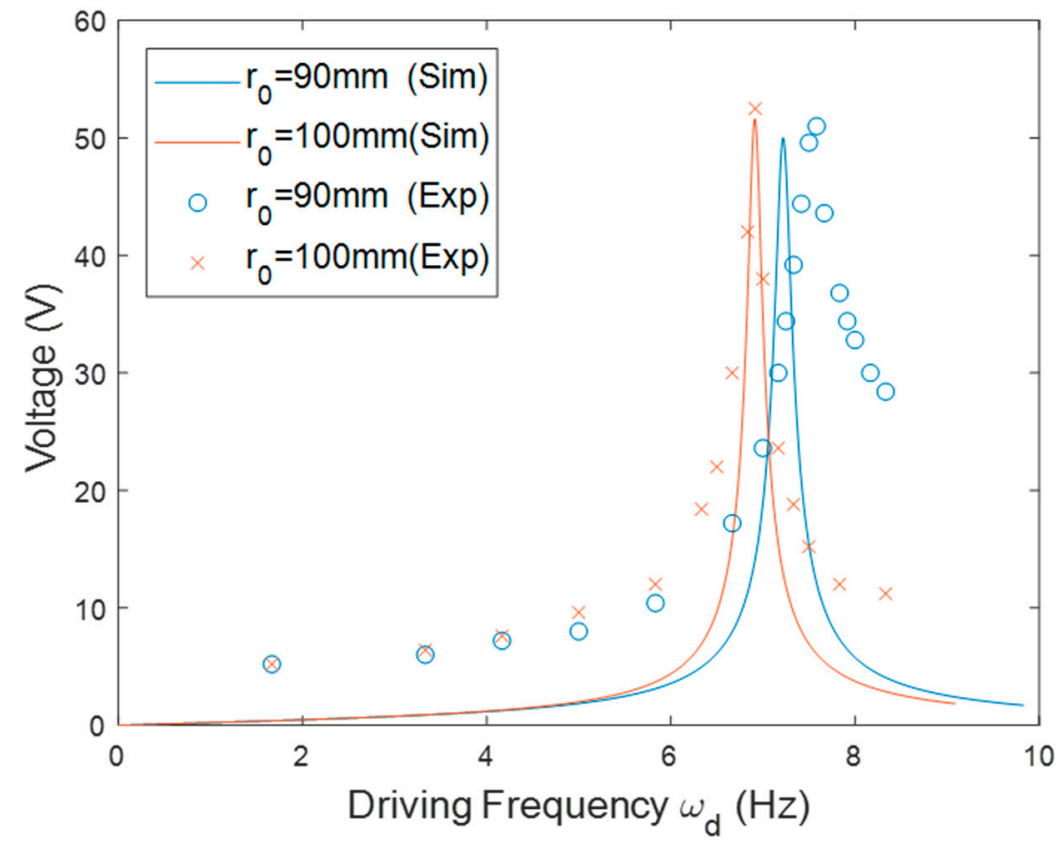

Figure 8. The simulation and experimental voltage responses of the $\mathrm{PEH}$ in the inward orientation with different $r_{0}$.

Finally, the resonant frequency of the PEH in the tilted configuration is illustrated in Figure 9. The length of the beam used in this configuration was $78 \mathrm{~mm}$ and $r_{0}$ was set to $30 \mathrm{~mm}$. The PEH was tested with different tilt angles. It was expected that the tilt angle would change the magnitude of the component of centrifugal force in the longitudinal direction of the beam. The result showed that the resonant frequency decreased as the tilt angle increased, because a large tilt angle reduced the tensile axial load caused by the centrifugal force. The slope of the curve became less steep when the angle increased. A similar trend of the resonant frequency was seen in both the simulation and the experimental results shown in Figure 10. The resonant frequency reduced as the tilt angle increased in the simulation and experimental results. The peak voltage was enhanced when the resonant frequency 
was decreased. Note that there was a mismatch between the numerical and experimental resonant frequency when the tilt angle was $90^{\circ}$. The reason for this is that the component of the centrifugal force in the transverse direction of the beam made the beam deflect. The deflection would strengthen the axial load, as indicated in Figure 11, since the deflection resulted in a larger component of the centrifugal force in the axial direction. Therefore, the experimental stiffness would be higher than the stiffness estimated by the model and consequently the experimental resonant frequency would be higher than the numerical one.

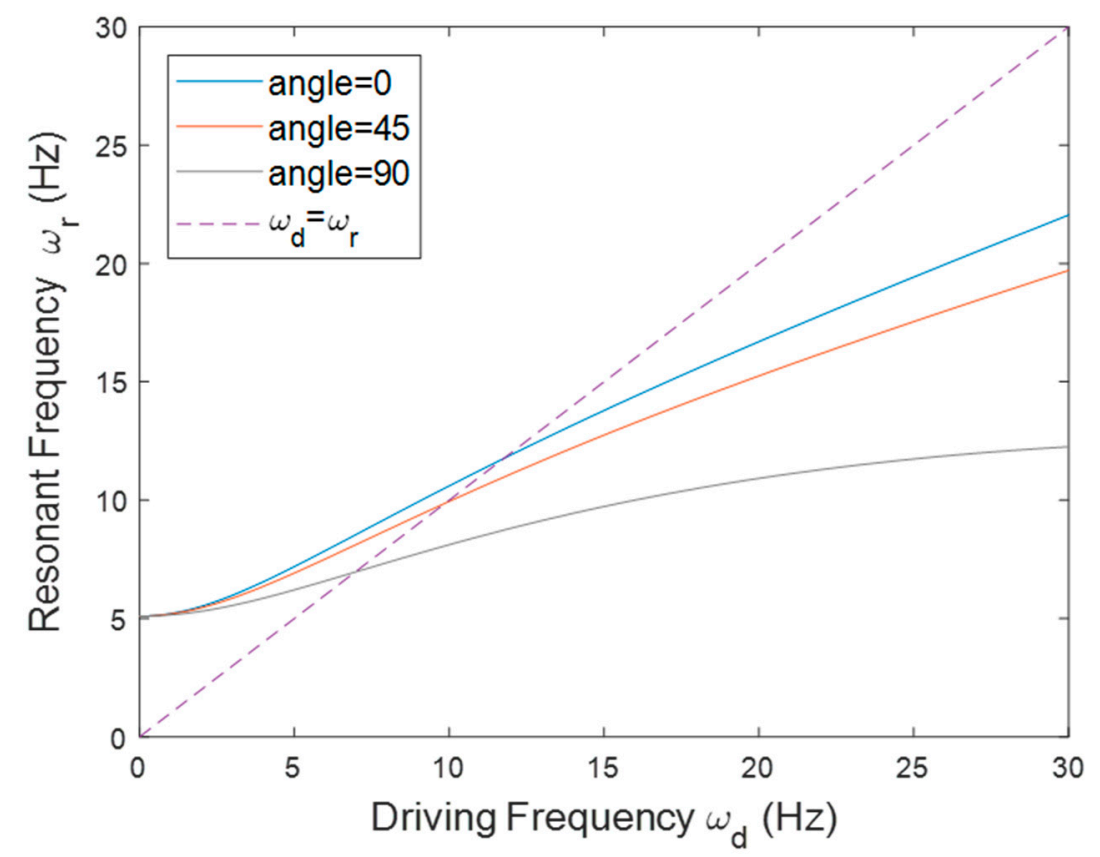

Figure 9. The resonant frequency of the PEH in the tilted configuration with different $\theta$.

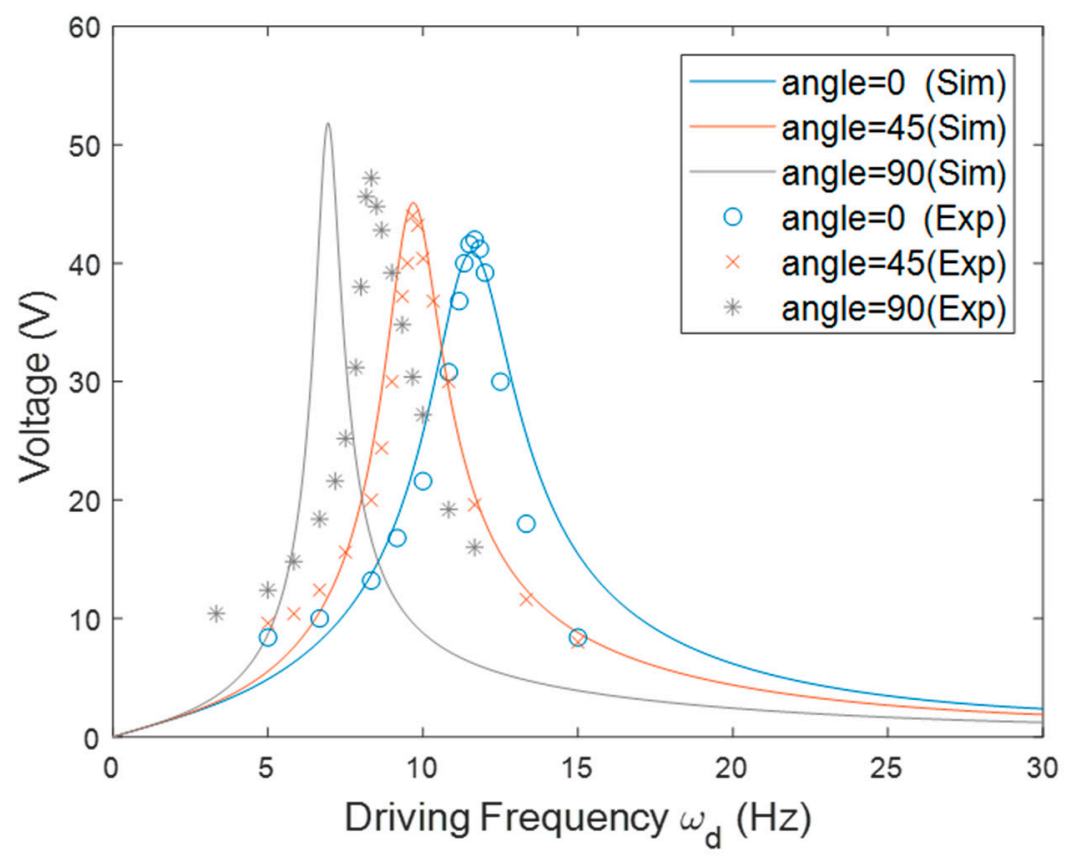

Figure 10. The simulation and experimental voltage responses of the PEH in the tilted orientation with different $\theta$. 


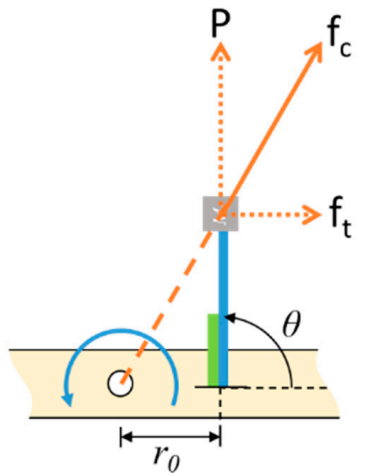

without deformation (model)

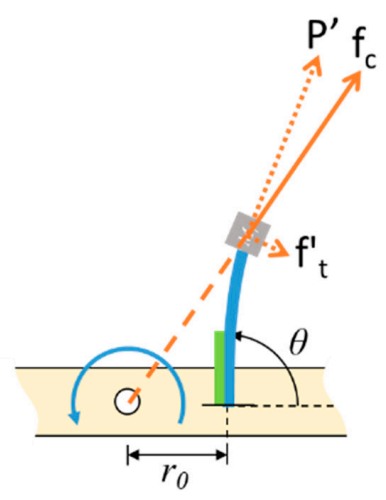

with deformation (experiment)

Figure 11. The illustration of axial load on the PEH in the tilted configuration.

\section{Conclusions}

This study examined a PEH in different orientations under rotational excitations. The installation orientation of the PEH influenced the axial component of the centrifugal force acting on the beam so the dynamics of the PEH were affected correspondingly. The dynamics of the rotational PEH was theoretically modeled and an experimental study was conducted for validation. Three configurations-outward, inward, and tilted orientations-were studied and compared. In the outward orientation, the resonant frequency of the PEH increased when the installation radius was extended, because of the increase of the centrifugal force. However, the experiment and simulation showed that the resonant frequency of the $\mathrm{PEH}$ in the inward orientation was raised as the installation radius was shortened. Finally, the tilt angle of the PEH had a strong influence on the resonant frequency of the $\mathrm{PEH}$. When the tilt angle increased from $0^{\circ}$ to $90^{\circ}$, the increase of the resonant frequency with respect to the driving frequency became less significant. In addition, as the tilt angle increased, the tensile axial load caused by the centrifugal force was reduced so the resonant frequency was lowered. Overall, this study shows that both the installation radius and the tilt angle could be used to tune the matching frequency to fit the environment for high power output. Although the installation radius was effective for tuning the matching frequency, it is constrained by the size of the rotating hub and sometimes cannot be freely adjusted. The tilt angle, however, can be used to adjust the matching frequency when the installation radius is limited.

Author Contributions: Conceptualization, W.-J.S. and J.-H.L.; methodology, W.-J.S. and J.-H.L.; software, J.-H.L.; validation, J.-H.L.; formal analysis, J.-H.L.; investigation, W.-J.S., J.-H.L. and W.-C.L.; resources, W.-J.S.; data curation, J.-H.L.; writing-original draft preparation, W.-J.S.; writing-review and editing, W.-J.S. and W.-C.L.; visualization, W.-J.S. and J.-H.L.; supervision, W.-J.S. and W.-C.L.; project administration, W.-J.S.; funding acquisition, W.-J.S. All authors have read and agreed to the published version of the manuscript.

Funding: This research was funded by the Ministry of Science and Technology of Taiwan, grant number MOST 107-2221-E-002-118-MY3. The APC was funded by the Ministry of Science and Technology of Taiwan.

Acknowledgments: The authors gratefully acknowledge the financial support of the Ministry of Science and Technology of Taiwan under grant number MOST 107-2221-E-002-118-MY3.

Conflicts of Interest: The authors declare no conflicts of interest.

\section{References}

1. Roundy, S.; Wright, P.K.; Rabaey, J. A study of low level vibrations as a power source for wireless sensor nodes. Comput. Commun. 2003, 26, 1131-1144. [CrossRef]

2. Roundy, S.; Wright, P.K. A piezoelectric vibration based generator for wireless electronics. Smart Mater. Struct. 2004, 13, 1131-1142. [CrossRef]

3. Reilly, E.K.; Burghardt, F.; Fain, R.; Wright, P. Powering a wireless sensor node with a vibration-driven piezoelectric energy harvester. Smart Mater. Struct. 2011, 20. [CrossRef] 
4. Ng, T.H.; Liao, W.H. Sensitivity Analysis and Energy Harvesting for a Self-Powered Piezoelectric Sensor. J. Intell. Mater. Syst. Struct. 2005, 16, 785-797. [CrossRef]

5. Amin Karami, M.; Inman, D.J. Powering pacemakers from heartbeat vibrations using linear and nonlinear energy harvesters. Appl. Phys. Lett. 2012, 100. [CrossRef]

6. Hwang, G.T.; Park, H.; Lee, J.H.; Oh, S.; Park, K.I.; Byun, M.; Park, H.; Ahn, G.; Jeong, C.K.; No, K.; et al. Self-powered cardiac pacemaker enabled by flexible single crystalline PMN-PT piezoelectric energy harvester. Adv. Mater. 2014, 26, 4880-4887. [CrossRef]

7. Paradiso, J.A.; Starner, T. Energy scavenging for mobile and wireless electronics. IEEE Pervasive Comput. 2005, 4, 18-27. [CrossRef]

8. Cook-Chennault, K.A.; Thambi, N.; Sastry, A.M. Powering MEMS portable devices-A review of non-regenerative and regenerative power supply systems with special emphasis on piezoelectric energy harvesting systems. Smart Mater. Struct. 2008, 17. [CrossRef]

9. Nechibvute, A.; Chawanda, A.; Luhanga, P. Piezoelectric Energy Harvesting Devices: An Alternative Energy Source for Wireless Sensors. Smart Mater. Res. 2012, 2012, 1-13. [CrossRef]

10. Erturk, A.; Inman, D.J. Issues in mathematical modeling of piezoelectric energy harvesters. Smart Mater. Struct. 2008, 17. [CrossRef]

11. Erturk, A.; Inman, D.J. A Distributed Parameter Electromechanical Model for Cantilevered Piezoelectric Energy Harvesters. J. Vib. Acoust. 2008, 130. [CrossRef]

12. Yang, Y.; Tang, L. Equivalent Circuit Modeling of Piezoelectric Energy Harvesters. J. Intell. Mater. Syst. Struct. 2009, 20, 2223-2235. [CrossRef]

13. Erturk, A.; Inman, D.J. On Mechanical Modeling of Cantilevered Piezoelectric Vibration Energy Harvesters. J. Intell. Mater. Syst. Struct. 2008, 19, 1311-1325. [CrossRef]

14. Dietl, J.M.; Wickenheiser, A.M.; Garcia, E. A Timoshenko beam model for cantilevered piezoelectric energy harvesters. Smart Mater. Struct. 2010, 19. [CrossRef]

15. Hu, Y.; Xue, H.; Hu, H. A piezoelectric power harvester with adjustable frequency through axial preloads. Smart Mater. Struct. 2007, 16, 1961-1966. [CrossRef]

16. Eichhorn, C.; Goldschmidtboeing, F.; Woias, P. Bidirectional frequency tuning of a piezoelectric energy converter based on a cantilever beam. J. Micromech. Microeng. 2009, 19. [CrossRef]

17. Al-Ashtari, W.; Hunstig, M.; Hemsel, T.; Sextro, W. Frequency tuning of piezoelectric energy harvesters by magnetic force. Smart Mater. Struct. 2012, 21. [CrossRef]

18. Dong, L.; Prasad, M.G.; Fisher, F.T. Two-dimensional resonance frequency tuning approach for vibration-based energy harvesting. Smart Mater. Struct. 2016, 25. [CrossRef]

19. Bouhedma, S.; Zheng, Y.; Lange, F.; Hohlfeld, D. Magnetic Frequency Tuning of a Multimodal Vibration Energy Harvester. Sensors 2019, 19, 1149. [CrossRef]

20. Morel, A.; Pillonnet, G.; Gasnier, P.; Lefeuvre, E.; Badel, A. Frequency tuning of piezoelectric energy harvesters thanks to a short-circuit synchronous electric charge extraction. Smart Mater. Struct. 2019, 28. [CrossRef]

21. Brenes, A.; Morel, A.; Gibus, D.; Yoo, C.S.; Gasnier, P.; Lefeuvre, E.; Badel, A. Large-bandwidth piezoelectric energy harvesting with frequency-tuning synchronized electric charge extraction. Sens. Actuators A Phys. 2020, 302. [CrossRef]

22. Xue, T.; Ma, X.; Rahn, C.; Roundy, S. Analysis of Upper Bound Power Output for a Wrist-Worn Rotational Energy Harvester from Real-World Measured Inputs. J. Phys. Conf. Ser. 2014, 557. [CrossRef]

23. Pillatsch, P.; Yeatman, E.M.; Holmes, A.S. A piezoelectric frequency up-converting energy harvester with rotating proof mass for human body applications. Sens. Actuators A Phys. 2014, 206, 178-185. [CrossRef]

24. Ramezanpour, R.; Nahvi, H.; Ziaei-Rad, S. Increasing the Performance of a Rotary Piezoelectric Frequency Up-Converting Energy Harvester Under Weak Excitations. J. Vib. Acoust. 2016, 139. [CrossRef]

25. Fu, H.; Yeatman, E.M. A methodology for low-speed broadband rotational energy harvesting using piezoelectric transduction and frequency up-conversion. Energy 2017, 125, 152-161. [CrossRef]

26. Shu, Y.C.; Wang, W.C.; Chang, Y.P. Electrically rectified piezoelectric energy harvesting induced by rotary magnetic plucking. Smart Mater. Struct. 2018, 27. [CrossRef]

27. Janphuang, P.; Lockhart, R.; Henein, S.; Briand, D.; de Rooij, N.F. On the experimental determination of the efficiency of piezoelectric impact-type energy harvesters using a rotational flywheel. J. Phys. Conf. Ser. 2013, 476. [CrossRef] 
28. Gu, L.; Livermore, C. Passive self-tuning energy harvester for extracting energy from rotational motion. Appl. Phys. Lett. 2010, 97, 081904. [CrossRef]

29. Hsu, J.-C.; Tseng, C.-T.; Chen, Y.-S. Analysis and experiment of self-frequency-tuning piezoelectric energy harvesters for rotational motion. Smart Mater. Struct. 2014, 23, 075013. [CrossRef]

30. Rui, X.; Zeng, Z.; Zhang, Y.; Li, Y.; Feng, H.; Huang, X.; Sha, Z. Design and Experimental Investigation of a Self-Tuning Piezoelectric Energy Harvesting System for Intelligent Vehicle Wheels. IEEE Trans. Veh. Technol. 2019. [CrossRef]

31. Guan, M.; Liao, W.-H. Design and analysis of a piezoelectric energy harvester for rotational motion system. Energy Convers. Manag. 2016, 111, 239-244. [CrossRef]

32. Gu, L.; Livermore, C. Compact passively self-tuning energy harvesting for rotating applications. Smart Mater. Struct. 2012, 21, 015002. [CrossRef]

33. Wang, Y.-J.; Chuang, T.-Y.; Yu, J.-H. Design and kinetic analysis of piezoelectric energy harvesters with self-adjusting resonant frequency. Smart Mater. Struct. 2017, 26, 095037. [CrossRef]

34. Hsieh, T.T.; Chen, S.A.; Shu, Y.-C. Investigation of various cantilever configurations for piezoelectric energy harvesting under rotational motion. In Proceedings of the SPIE 10967, Active and Passive Smart Structures and Integrated Systems XIII, Denver, CO, USA, 21 March 2019; p. 1096719.

35. Sadeqi, S.; Arzanpour, S.; Hajikolaei, K.H. Broadening the Frequency Bandwidth of a Tire-Embedded Piezoelectric-Based Energy Harvesting System Using Coupled Linear Resonating Structure. IEEE/ASME Trans. Mechatron. 2015, 20, 2085-2094. [CrossRef]

36. Febbo, M.; Machado, S.P.; Gatti, C.D.; Ramirez, J.M. An out-of-plane rotational energy harvesting system for low frequency environments. Energy Convers. Manag. 2017, 152, 166-175. [CrossRef]

37. Ramírez, J.M.; Gatti, C.D.; Machado, S.P.; Febbo, M. A piezoelectric energy harvester for rotating environment using a linked E-shape multi-beam. Extrem. Mech. Lett. 2019, 27, 8-19. [CrossRef]

38. Zhang, Y.; Zheng, R.; Kaizuka, T.; Su, D.; Nakano, K.; Cartmell, M.P. Broadband vibration energy harvesting by application of stochastic resonance from rotational environments. Eur. Phys. J. Spec. Top. 2015, 224, 2687-2701. [CrossRef]

39. Guo, B.; Chen, Z.; Cheng, C.; Yang, Y. Characteristics of a nonlinear rotating piezoelectric energy harvester under variable rotating speeds. Int. J. Appl. Electromagn. Mech. 2015, 47, 411-423. [CrossRef]

40. Chen, Z.; Guo, B.; Xiong, Y.; Cheng, C.; Yang, Y. Melnikov-method-based broadband mechanism and necessary conditions of nonlinear rotating energy harvesting using piezoelectric beam. J. Intell. Mater. Syst. Struct. 2016, 27, 2555-2567. [CrossRef]

41. Cheng, C.; Chen, Z.; Xiong, Y.; Shi, H.; Yang, Y. A high-efficiency, self-powered nonlinear interface circuit for bi-stable rotating piezoelectric vibration energy harvesting with nonlinear magnetic force. Int. J. Appl. Electromagn. Mech. 2016, 51, 235-248. [CrossRef]

42. Zou, H.-X.; Zhang, W.-M.; Li, W.-B.; Wei, K.-X.; Gao, Q.-H.; Peng, Z.-K.; Meng, G. Design and experimental investigation of a magnetically coupled vibration energy harvester using two inverted piezoelectric cantilever beams for rotational motion. Energy Convers. Manag. 2017, 148, 1391-1398. [CrossRef]

(C) 2020 by the authors. Licensee MDPI, Basel, Switzerland. This article is an open access article distributed under the terms and conditions of the Creative Commons Attribution (CC BY) license (http://creativecommons.org/licenses/by/4.0/). 